\title{
Federer's characterization of sets of finite perimeter in metric spaces *
}

\author{
Panu Lahti
}

May 1, 2018

\begin{abstract}
Federer's characterization of sets of finite perimeter states (in Euclidean spaces) that a set is of finite perimeter if and only if the measure-theoretic boundary of the set has finite Hausdorff measure of codimension one. In complete metric spaces that are equipped with a doubling measure and support a Poincaré inequality, the "only if" direction was shown by Ambrosio (2002). By applying fine potential theory in the case $p=1$, we prove that the "if" direction holds as well.
\end{abstract}

\section{Introduction}

In the past two decades, there has been great interest in studying problems of firstorder analysis in the setting of general metric measure spaces, see e.g. [2, 4, 5, 14, 29, 30. In particular, Sobolev functions (sometimes called Newton-Sobolev functions in the metric setting) and functions of bounded variation (BV functions) have been topics of central interest. In much of the literature (as well as in the current paper) one assumes that the space is complete, equipped with a doubling measure, and supports a Poincaré inequality; see Section 2 for definitions. Studying questions in such an abstract setting provides an opportunity to unify the theories developed in specific settings such as weighted Euclidean spaces, Riemannian manifolds, Carnot groups, etc. Moreover, without having the Euclidean structure available, one is forced to develop novel methods and proofs, giving new insight into various problems.

In the theory of BV functions in the Euclidean setting, a key result originally due to De Giorgi states that if $E$ is a set of finite perimeter, then the perimeter measure $P(E, \cdot)$ coincides with the $n$-1-dimensional Hausdorff measure restricted to the measure-theoretic boundary $\partial^{*} E$. In particular, $P\left(E, \mathbb{R}^{n}\right)<\infty$ implies $\mathcal{H}^{n-1}\left(\partial^{*} E\right)<\infty$. By a deep result due to Federer [11, Section 4.5.11], the converse holds as well, so in fact $P\left(E, \mathbb{R}^{n}\right)<\infty$ if and only if $\mathcal{H}^{n-1}\left(\partial^{*} E\right)<\infty$. This is known as Federer's characterization of sets of finite perimeter. In the metric setting,

*2010 Mathematics Subject Classification: 30L99, 31E05, 26 B30.

Keywords: metric measure space, set of finite perimeter, Federer's characterization, measuretheoretic boundary, codimension one Hausdorff measure, fine topology. 
where it is natural to formulate this kind of result by means of the codimension one Hausdorff measure $\mathcal{H}$, the "only if" direction of the characterization was shown by Ambrosio [2], but the "if" direction has remained an open problem. In the current paper, we show that this direction holds as well.

Theorem 1.1. Let $\Omega \subset X$ be an open set, let $E \subset X$ be a $\mu$-measurable set, and suppose that $\mathcal{H}\left(\partial^{*} E \cap \Omega\right)<\infty$. Then $P(E, \Omega)<\infty$.

The "only if" direction of Federer's characterization is part of a more general structure theorem for sets of finite perimeter, which in the metric setting states that the perimeter measure is comparable to the Hausdorff measure of codimension one restricted to the measure-theoretic boundary. This structure theorem is an indispensable tool in analysis of sets of finite perimeter, and hence more general BV functions as well. While not equally essential, the "if" direction of Federer's characterization has a number of applications as well. For example, in [16] the authors proved a characterization of Newton-Sobolev functions with zero boundary values by means of a natural Lebesgue point-type condition on the boundary. However, the proof relied on assuming that Federer's characterization holds; now we know that this is the case under the usual assumptions on the space. We will discuss other applications in Section 5 .

Previously there have been some partial results toward a proof of the "if" direction. The paper [19] showed that if the metric space is assumed to contain a "thick" bundle of curves between each pair of points, then the "if" direction can be proved by mimicking the Euclidean proof. In the current paper we take a completely different approach, which relies on fine potential theory. In the case $1<p<\infty$, fine potential theory deals with superharmonic functions as understood by means of the fine topology; see the monographs [1, 13, 28, for the theory and its history in the Euclidean setting, and the recent papers [6, 7, 8, 9] for similar results in the metric setting. In [21, the author proved some analogous results in the case $p=1$, by relying on certain continuity properties of BV functions proved earlier in [22, 25]. An application of these results led to following characterization of sets of finite perimeter, which is in the same vein as Federer's characterization. Below, $\partial^{1} I_{E}$ denotes the fine boundary of $E$, or more precisely of its measure-theoretic interior; one always has $\partial^{*} E \subset \partial^{1} I_{E}$.

Theorem 1.2 ([21, Theorem 1.1]). For an open set $\Omega \subset X$ and a $\mu$-measurable set $E \subset X$, we have $P(E, \Omega)<\infty$ if and only if $\mathcal{H}\left(\partial^{1} I_{E} \cap \Omega\right)<\infty$. Furthermore, then $\mathcal{H}\left(\left(\partial^{1} I_{E} \backslash \partial^{*} E\right) \cap \Omega\right)=0$.

In the current paper, our main goal is to show that if $\mathcal{H}\left(\partial^{*} E \cap \Omega\right)<\infty$, then $\mathcal{H}\left(\left(\partial^{1} I_{E} \backslash \partial^{*} E\right) \cap \Omega\right)=0$ and thus Theorem 1.1 follows from Theorem 1.2. The proofs will be given in Section 4, and they rely mostly on properties of the 1-fine topology proved in [21, 23], as well as boxing inequality-type arguments. Our methods and the underlying theory should be of interest already in Euclidean spaces, where Federer's original argument has remained (as far as we know) essentially the only known proof for the characterization. 
Acknowledgments. The author wishes to thank Nageswari Shanmugalingam and Juha Kinnunen for reading the manuscript and giving comments that helped improve the paper.

\section{Preliminaries}

In this section we introduce the standard definitions, notation, and assumptions used in the paper.

Throughout this paper, $(X, d, \mu)$ is a complete metric space that is equipped with a metric $d$ and a Borel regular outer measure $\mu$ satisfying a doubling property, meaning that there exists a constant $C_{d} \geq 1$ such that

$$
0<\mu(B(x, 2 r)) \leq C_{d} \mu(B(x, r))<\infty
$$

for every ball $B(x, r):=\{y \in X: d(y, x)<r\}$. We assume that $X$ consists of at least 2 points. Given a ball $B=B(x, r)$ and $\beta>0$, we sometimes abbreviate $\beta B:=B(x, \beta r)$. Note that in metric spaces, a ball (as a set) does not necessarily have a unique center point and radius, but we understand these to be prescribed for all balls that we consider. When we want to state that a constant $C$ depends on the parameters $a, b, \ldots$, we write $C=C(a, b, \ldots)$.

All functions defined on $X$ or its subsets will take values in $[-\infty, \infty]$. A complete metric space equipped with a doubling measure is proper, that is, closed and bounded sets are compact. For any open set $\Omega \subset X$, we $\operatorname{define}_{\operatorname{Lip}}(\Omega)$ as the set of functions that are in the class $\operatorname{Lip}\left(\Omega^{\prime}\right)$ for every open $\Omega^{\prime} \Subset \Omega$; here $\Omega^{\prime} \Subset \Omega$ means that $\overline{\Omega^{\prime}}$ is a compact subset of $\Omega$. Other local function spaces are defined analogously.

For any set $A \subset X$ and $0<R<\infty$, the restricted spherical Hausdorff content of codimension one is defined as

$$
\mathcal{H}_{R}(A):=\inf \left\{\sum_{i=1}^{\infty} \frac{\mu\left(B\left(x_{i}, r_{i}\right)\right)}{r_{i}}: A \subset \bigcup_{i=1}^{\infty} B\left(x_{i}, r_{i}\right), r_{i} \leq R\right\} .
$$

The codimension one Hausdorff measure of $A \subset X$ is then defined as

$$
\mathcal{H}(A):=\lim _{R \rightarrow 0} \mathcal{H}_{R}(A) .
$$

By a curve we mean a nonconstant rectifiable continuous mapping from a compact interval of the real line into $X$. A nonnegative Borel function $g$ on $X$ is an upper gradient of a function $u$ on $X$ if for all curves $\gamma$, we have

$$
|u(x)-u(y)| \leq \int_{\gamma} g d s,
$$

where $x$ and $y$ are the end points of $\gamma$ and the curve integral is defined by using an arc-length parametrization, see [14, Section 2] where upper gradients were originally introduced. We interpret $|u(x)-u(y)|=\infty$ whenever at least one of $|u(x)|,|u(y)|$ is infinite. 
Let $1 \leq p<\infty$ (we will work almost exclusively with $p=1$ ). We say that a family of curves $\Gamma$ is of zero $p$-modulus if there is a nonnegative Borel function $\rho \in L^{p}(X)$ such that for all curves $\gamma \in \Gamma$, the curve integral $\int_{\gamma} \rho d s$ is infinite. A property is said to hold for $p$-almost every curve if it fails only for a curve family with zero $p$-modulus. If $g$ is a nonnegative $\mu$-measurable function on $X$ and (2.1) holds for $p$-almost every curve, we say that $g$ is a $p$-weak upper gradient of $u$. By only considering curves $\gamma$ in a set $A \subset X$, we can talk about a function $g$ being a ( $p$-weak) upper gradient of $u$ in $A$.

Given an open set $\Omega \subset X$, we let

$$
\|u\|_{N^{1, p}(\Omega)}:=\|u\|_{L^{p}(\Omega)}+\inf \|g\|_{L^{p}(\Omega)},
$$

where the infimum is taken over all $p$-weak upper gradients $g$ of $u$ in $\Omega$. The substitute for the Sobolev space $W^{1, p}$ in the metric setting is the Newton-Sobolev space

$$
N^{1, p}(\Omega):=\left\{u:\|u\|_{N^{1, p}(\Omega)}<\infty\right\},
$$

which was introduced in [30]. We understand a Newton-Sobolev function to be defined at every $x \in \Omega$ (even though $\|\cdot\|_{N^{1, p}(\Omega)}$ is then only a seminorm). It is known that for any $u \in N_{\text {loc }}^{1, p}(\Omega)$ there exists a minimal $p$-weak upper gradient of $u$ in $\Omega$, always denoted by $g_{u}$, satisfying $g_{u} \leq g$ almost everywhere in $\Omega$, for any $p$-weak upper gradient $g \in L_{\mathrm{loc}}^{p}(\Omega)$ of $u$ in $\Omega$, see [5, Theorem 2.25].

The space of Newton-Sobolev functions with zero boundary values is defined as

$$
N_{0}^{1, p}(\Omega):=\left\{\left.u\right|_{\Omega}: u \in N^{1, p}(X) \text { and } u=0 \text { on } X \backslash \Omega\right\} .
$$

This class can be understood to be a subclass of $N^{1, p}(X)$ in a natural way.

The $p$-capacity of a set $A \subset X$ is defined as

$$
\operatorname{Cap}_{p}(A):=\inf \|u\|_{N^{1, p}(X)},
$$

where the infimum is taken over all functions $u \in N^{1, p}(X)$ such that $u \geq 1$ in $A$.

The variational 1-capacity of a set $A \subset \Omega$ with respect to an open set $\Omega \subset X$ is defined as

$$
\operatorname{cap}_{1}(A, \Omega):=\inf \int_{X} g_{u} d \mu
$$

where the infimum is taken over functions $u \in N_{0}^{1,1}(\Omega)$ such that $u \geq 1$ on $A$, and $g_{u}$ is the minimal 1-weak upper gradient of $u$ (in $X$ ). For basic properties satisfied by capacities, such as monotonicity and countable subadditivity, see [5].

We will assume throughout the paper that $X$ supports a $(1,1)$-Poincaré inequality, meaning that there exist constants $C_{P}>0$ and $\lambda \geq 1$ such that for every ball $B(x, r)$, every $u \in L_{\text {loc }}^{1}(X)$, and every upper gradient $g$ of $u$, we have

$$
f_{B(x, r)}\left|u-u_{B(x, r)}\right| d \mu \leq C_{P} r f_{B(x, \lambda r)} g d \mu,
$$

where

$$
u_{B(x, r)}:=f_{B(x, r)} u d \mu:=\frac{1}{\mu(B(x, r))} \int_{B(x, r)} u d \mu .
$$


Next we recall the definition and basic properties of functions of bounded variation on metric spaces, following [29. See also e.g. [3, 10, 11, 12, 31, for the classical theory in the Euclidean setting. Let $\Omega \subset X$ be an open set. Given a function $u \in L_{\text {loc }}^{1}(\Omega)$, we define the total variation of $u$ in $\Omega$ as

$$
\|D u\|(\Omega):=\inf \left\{\liminf _{i \rightarrow \infty} \int_{\Omega} g_{u_{i}} d \mu: u_{i} \in N_{\mathrm{loc}}^{1,1}(\Omega), u_{i} \rightarrow u \text { in } L_{\mathrm{loc}}^{1}(\Omega)\right\},
$$

where each $g_{u_{i}}$ is the minimal 1-weak upper gradient of $u_{i}$ in $\Omega$. (In [29], local Lipschitz constants were used instead of upper gradients, but the properties of the total variation can be proved similarly with either definition.) We say that a function $u \in L^{1}(\Omega)$ is of bounded variation, and denote $u \in \operatorname{BV}(\Omega)$, if $\|D u\|(\Omega)<\infty$. For an arbitrary set $A \subset X$, we define

$$
\|D u\|(A):=\inf \{\|D u\|(W): A \subset W, W \subset X \text { is open }\} .
$$

If $u \in L_{\text {loc }}^{1}(\Omega)$ and $\|D u\|(\Omega)<\infty,\|D u\|(\cdot)$ is a Radon measure on $\Omega$ by [29, Theorem 3.4]. A $\mu$-measurable set $E \subset X$ is said to be of finite perimeter if $\left\|D \chi_{E}\right\|(X)<\infty$, where $\chi_{E}$ is the characteristic function of $E$. The perimeter of $E$ in $\Omega$ is also denoted by

$$
P(E, \Omega):=\left\|D \chi_{E}\right\|(\Omega) .
$$

Applying the Poincaré inequality to sequences of approximating locally Lipschitz functions in the definition of the total variation, we get the following $\mathrm{BV}$ version: for every ball $B(x, r)$ and every $u \in L_{\mathrm{loc}}^{1}(X)$, we have

$$
f_{B(x, r)}\left|u-u_{B(x, r)}\right| d \mu \leq C_{P} r \frac{\|D u\|(B(x, \lambda r))}{\mu(B(x, \lambda r))} .
$$

For a $\mu$-measurable set $E \subset X$, this implies the relative isoperimetric inequality

$$
\min \{\mu(B(x, r) \cap E), \mu(B(x, r) \backslash E)\} \leq 2 C_{P} r P(E, B(x, \lambda r)) ;
$$

see e.g. 18, Equation (3.1)].

The measure-theoretic interior of a set $E \subset X$ is defined as

$$
I_{E}:=\left\{x \in X: \lim _{r \rightarrow 0} \frac{\mu(B(x, r) \backslash E)}{\mu(B(x, r))}=0\right\},
$$

and the measure-theoretic exterior as

$$
O_{E}:=\left\{x \in X: \lim _{r \rightarrow 0} \frac{\mu(B(x, r) \cap E)}{\mu(B(x, r))}=0\right\} .
$$

The measure-theoretic boundary $\partial^{*} E$ is defined as the set of points $x \in X$ at which both $E$ and its complement have strictly positive upper density, i.e.

$$
\limsup _{r \rightarrow 0} \frac{\mu(B(x, r) \cap E)}{\mu(B(x, r))}>0 \quad \text { and } \quad \limsup _{r \rightarrow 0} \frac{\mu(B(x, r) \backslash E)}{\mu(B(x, r))}>0 .
$$


Then $X=I_{E} \cup O_{E} \cup \partial^{*} E$.

For an open set $\Omega \subset X$ and a $\mu$-measurable set $E \subset X$ with $P(E, \Omega)<\infty$, we know that for any Borel set $A \subset \Omega$,

$$
P(E, A)=\int_{\partial^{*} E \cap A} \theta_{E} d \mathcal{H}
$$

where $\theta_{E}: \partial^{*} E \rightarrow\left[\alpha, C_{d}\right]$ with $\alpha=\alpha\left(C_{d}, C_{P}, \lambda\right)>0$, see [2, Theorem 5.3] and [4, Theorem 4.6].

If $\Omega \subset X$ is an open set and $u, v \in L_{\text {loc }}^{1}(\Omega)$, then

$$
\|D \min \{u, v\}\|(\Omega)+\|D \max \{u, v\}\|(\Omega) \leq\|D u\|(\Omega)+\|D v\|(\Omega) ;
$$

for a proof see e.g. [24, Lemma 3.1].

The lower and upper approximate limits of a function $u$ on $X$ are defined respectively by

$$
u^{\wedge}(x):=\sup \left\{t \in \mathbb{R}: \lim _{r \rightarrow 0} \frac{\mu(B(x, r) \cap\{u<t\})}{\mu(B(x, r))}=0\right\}
$$

and

$$
u^{\vee}(x):=\inf \left\{t \in \mathbb{R}: \lim _{r \rightarrow 0} \frac{\mu(B(x, r) \cap\{u>t\})}{\mu(B(x, r))}=0\right\} .
$$

Unlike Newton-Sobolev functions, we understand BV functions to be $\mu$-equivalence classes. To consider fine properties, we need to consider the pointwise representatives $u^{\wedge}$ and $u^{\vee}$. We note that for $u=\chi_{E}$ with $E \subset X$, we have $x \in I_{E}$ if and only if $u^{\wedge}(x)=u^{\vee}(x)=1, x \in O_{E}$ if and only if $u^{\wedge}(x)=u^{\vee}(x)=0$, and $x \in \partial^{*} E$ if and only if $u^{\wedge}(x)=0$ and $u^{\vee}(x)=1$.

Throughout this paper we assume that $(X, d, \mu)$ is a complete metric space that is equipped with the doubling measure $\mu$ and supports a $(1,1)$-Poincaré inequality.

\section{The 1-fine topology}

In this section we have gathered all the results concerning the 1-fine topology that our argument will rely on. For these, we refer to [21, 22, 23]. Most of the results are analogous to those that hold in the case $1<p<\infty$, which has been studied in the metric setting in [6, 8, 9].

Definition 3.1. We say that $A \subset X$ is 1-thin at the point $x \in X$ if

$$
\lim _{r \rightarrow 0} r \frac{\operatorname{cap}_{1}(A \cap B(x, r), B(x, 2 r))}{\mu(B(x, r))}=0 .
$$

We also say that a set $U \subset X$ is 1-finely open if $X \backslash U$ is 1-thin at every $x \in U$. Then we define the 1-fine topology as the collection of 1-finely open sets on $X$.

We denote the 1-fine interior of a set $H \subset X$, i.e. the largest 1-finely open set contained in $H$, by fine-int $H$. We denote the 1-fine closure of a set $H \subset X$, i.e. 
the smallest 1-finely closed set containing $H$, by $\bar{H}^{1}$. The 1-fine boundary of $H$ is $\partial^{1} H:=\bar{H}^{1} \backslash$ fine-int $H$. Finally, the 1 -base $b_{1} H$ is defined as the set of points where $H$ is not 1-thin.

See [22, Section 4] for discussion on this definition, and for a proof of the fact that the 1-fine topology is indeed a topology. By [5, Proposition 6.16], a set $A \subset X$ is 1 -thin at $x \in X$ if and only if

$$
\lim _{r \rightarrow 0} \frac{\operatorname{cap}_{1}(A \cap B(x, r), B(x, 2 r))}{\operatorname{cap}_{1}(B(x, r), B(x, 2 r))}=0,
$$

and so it is clear that $W \subset b_{1} W$ for any open set $W \subset X$.

Now we collect some facts concerning the 1-fine topology proved in [21]. According to [21, Corollary 3.5], the 1-fine closure of $A \subset X$ can be characterized in the following way:

$$
\bar{A}^{1}=A \cup b_{1} A \text {. }
$$

From this it easily follows that for any $A \subset X$ and any ball $B(x, r)$, we have $\bar{A}^{1} \cap B(x, r) \subset \overline{A \cap B(x, r)}^{1}$, and then by [21, Proposition 3.3] we get

$$
\operatorname{cap}_{1}\left(\bar{A}^{1} \cap B(x, r), B(x, 2 r)\right)=\operatorname{cap}_{1}(A \cap B(x, r), B(x, 2 r)) .
$$

By [21, Lemma 4.6] the 1-fine boundary of a measure-theoretic interior can be characterized as follows: for any $\mu$-measurable set $E \subset X$,

$$
\partial^{1} I_{E}=b_{1} I_{E} \cap b_{1}\left(X \backslash I_{E}\right) .
$$

By [21, Lemma 3.1] we know that for any $\mu$-measurable set $E \subset X$,

$$
\partial^{*} E \subset \partial^{1} I_{E}
$$

Conversely, if $\Omega \subset X$ is open and $E \subset X$ is $\mu$-measurable such that $P(E, \Omega)<\infty$, then by Theorem 1.2 ,

$$
\mathcal{H}\left(\left(\partial^{1} I_{E} \backslash \partial^{*} E\right) \cap \Omega\right)=0 .
$$

Combining this with (2.4) gives

$$
\alpha \mathcal{H}\left(\partial^{1} I_{E} \cap \Omega\right) \leq P(E, \Omega) \leq C_{d} \mathcal{H}\left(\partial^{1} I_{E} \cap \Omega\right) .
$$

In fact this holds for every $\mu$-measurable $E \subset X$; to see this we can assume that $\mathcal{H}\left(\partial^{1} I_{E} \cap \Omega\right)<\infty$, and then $P(E, \Omega)<\infty$ by Theorem 1.2 .

We also have the following version of the relative isoperimetric inequality: for every ball $B(x, r)$ and every $\mu$-measurable $E \subset X$,

$$
\min \{\mu(B(x, r) \cap E), \mu(B(x, r) \backslash E)\} \leq 2 C_{P} C_{d} r \mathcal{H}\left(\partial^{1} I_{E} \cap B(x, \lambda r)\right) ;
$$

this follows from the ordinary relative isoperimetric inequality (2.2) and (3.6). 
Remark 3.8. It may seem strange to talk about $\partial^{1} I_{E}$, as it seems that we are first taking the interior in one topology and then the boundary in another. However, if we define the measure topology more axiomatically, then $I_{E}$ is actually not the interior of $E$ in the measure topology, and should be seen as a measure-theoretic quantity rather than a topological one (see [21, Remark 4.9]). Moreover, $\partial^{*} E$ is actually the boundary of $I_{E}$ in the measure topology; let us denote it by $\partial^{0} I_{E}$. Thus $\partial^{1} I_{E}$ is a natural set to consider as well. Finally, we can note that $\partial^{1} I_{E}=\partial^{1} O_{E}$, see [21, Lemma 4.8].

The following weak Cartan property in the case $p=1$ was proved in [23]. Note that here we have not defined the concept of 1-superminimizers, but we will not need it at all in this paper.

Theorem 3.9 ([23, Theorem 5.2]). Let $A \subset X$ and let $x \in X \backslash A$ be such that $A$ is 1-thin at $x$. Then there exist $R>0$ and $E_{0}, E_{1} \subset X$ such that $\chi_{E_{0}}, \chi_{E_{1}} \in \mathrm{BV}(X)$, $\chi_{E_{0}}$ and $\chi_{E_{1}}$ are 1-superminimizers in $B(x, R), \max \left\{\chi_{E_{0}}^{\wedge}, \chi_{E_{1}}^{\wedge}\right\}=1$ in $A \cap B(x, R)$, $\chi_{E_{0}}^{\vee}(x)=0=\chi_{E_{1}}^{\vee}(x),\left\{\max \left\{\chi_{E_{0}}^{\vee}, \chi_{E_{1}}^{\vee}\right\}>0\right\}$ is 1-thin at $x$, and

$$
\lim _{r \rightarrow 0} r \frac{P\left(E_{0}, B(x, r)\right)}{\mu(B(x, r))}=0, \quad \lim _{r \rightarrow 0} r \frac{P\left(E_{1}, B(x, r)\right)}{\mu(B(x, r))}=0 .
$$

The following simpler formulation will be sufficient for our purposes.

Corollary 3.11. Let $A \subset X$ and let $x \in X \backslash A$ be such that $A$ is 1 -thin at $x$. Then there exist $R>0$ and $F \subset X$ such that $\chi_{F} \in \mathrm{BV}(X), A \cap B(x, R) \subset I_{F}, I_{F}$ is 1-thin at $x$, and

$$
\lim _{r \rightarrow 0} r \frac{P(F, B(x, r))}{\mu(B(x, r))}=0 .
$$

Proof. Take $E_{0}, E_{1} \subset X$ as given by Theorem 3.9, and set $F:=E_{0} \cup E_{1}$. By (2.5) we obtain $\chi_{F} \in \mathrm{BV}(X)$, and (2.5) and (3.10) together give (3.12). From the fact that $\max \left\{\chi_{E_{0}}^{\wedge}, \chi_{E_{1}}^{\wedge}\right\}=1$ in $A \cap B(x, R)$ we obtain that

$$
A \cap B(x, R) \subset I_{E_{0}} \cup I_{E_{1}} \subset I_{F} .
$$

Finally, since $\left\{\max \left\{\chi_{E_{0}}^{\vee}, \chi_{E_{1}}^{\vee}\right\}>0\right\}$ is 1-thin at $x$, then so is

$$
I_{E_{0}} \cup I_{E_{1}} \cup \partial^{*} E_{0} \cup \partial^{*} E_{1} \supset I_{F} .
$$

In [23, Lemma 4.4] it was also shown that if $A \subset X$ is 1 -thin at a point $x \in X \backslash A$, then there exists an open set that contains $A$ and is also 1 -thin at $x$, that is,

If $x \notin A \cup b_{1} A$, then there exists an open $W \supset A$ such that $x \notin b_{1} W$. 


\section{Proof of the characterization}

In [18, Theorem 3.11] it was shown that for any $\mu$-measurable set $E \subset X$, we have $\overline{\partial^{*} E}=\partial I_{E}$, that is, the closure of the measure-theoretic boundary (in the metric topology) is the whole topological boundary of a suitable representative of $E$ (namely the measure-theoretic interior $I_{E}$ ). Now we prove the analogous result with the metric topology replaced by the 1-fine topology. This will be the crux of our proof of Federer's characterization.

Theorem 4.1. For any $\mu$-measurable set $E \subset X$, we have $\overline{\partial *}^{1}=\partial^{1} I_{E}$.

Note that by Remark 3.8, the above can be written as ${\overline{\partial^{0} I_{E}}}^{1}=\partial^{1} I_{E}$, showing that the result describes the interplay between the measure topology and the 1fine topology. It is natural to ask which other sets and topologies would satisfy an analogous property, but we will not pursue this problem here. Previously, properties of the measure topology and fine topologies have been studied in the monograph [27.

Proof. By (3.5) we have

$$
\overline{\partial *}^{1} \subset{\overline{\partial^{1} I_{E}}}^{1}=\partial^{1} I_{E},
$$

where the last equality follows from the fact that boundaries are closed sets in every topology. Thus we only need to show that $\bar{\partial}^{*} E^{1} \supset \partial^{1} I_{E}$. Let $x_{0} \in \partial^{1} I_{E}$ and let $U \ni x_{0}$ be a 1-finely open set. We need to show that $\partial^{*} E \cap U \neq \emptyset$. By (3.13) there exists an open set $W \supset X \backslash U$ that is 1-thin at $x_{0}$. Since $\bar{W}^{1}=W \cup b_{1} W=b_{1} W$ by (3.2), we have $x_{0} \notin \bar{W}^{1}$. We will show that $\partial^{*} E \backslash W \neq \emptyset$; suppose that instead $\partial^{*} E \backslash W=\emptyset$.

Claim. Let $x \in \partial^{1} I_{E} \backslash \bar{W}^{1}$ and $s_{1}>0$. Then there exists $y \in B\left(x, s_{1}\right) \cap \partial^{1} I_{E} \backslash \bar{W}^{1}$ and $0<s_{2} \leq s_{1} / 2$ such that

$$
\frac{1}{2 C_{d}^{\left\lceil\log _{2}(3 \lambda)\right\rceil+1}} \leq \frac{\mu\left(E \cap B\left(y, s_{2}\right)\right)}{\mu\left(B\left(y, s_{2}\right)\right)} \leq 1-\frac{1}{2 C_{d}^{\left\lceil\log _{2}(3 \lambda)\right\rceil}},
$$

where $\lceil a\rceil$ is the smallest integer at least $a \in \mathbb{R}$.

\section{Proof of claim:}

Step 1. We can assume that $x \in O_{E}$; the case $x \in I_{E}$ is handled analogously (recall that $\partial^{1} I_{E}=\partial^{1} O_{E}$ from Remark 3.8). Since $x \in \partial^{1} I_{E}$, by (3.4) we have

$$
\limsup _{r \rightarrow 0} r \frac{\operatorname{cap}_{1}\left(I_{E} \cap B(x, r), B(x, 2 r)\right)}{\mu(B(x, r))}>0 .
$$

Since $x$ belongs to the 1 -finely open set $X \backslash \bar{W}^{1}$, we have

$$
\lim _{r \rightarrow 0} r \frac{\operatorname{cap}_{1}\left(\bar{W}^{1} \cap B(x, r), B(x, 2 r)\right)}{\mu(B(x, r))}=0 .
$$


We apply Corollary 3.11 to find $R>0$ and $F \subset X$ such that $I_{F} \supset \bar{W}^{1} \cap B(x, R)$ and $I_{F}$ is 1 -thin at $x$. Then by (3.3), also

$$
\lim _{r \rightarrow 0} r \frac{\operatorname{cap}_{1}\left({\overline{I_{F}}}^{1} \cap B(x, r), B(x, 2 r)\right)}{\mu(B(x, r))}=0 .
$$

Combining this with (4.2), by subadditivity of the variational 1-capacity we get

$$
\limsup _{r \rightarrow 0} r \frac{\operatorname{cap}_{1}\left(\left(I_{E} \backslash{\overline{I_{F}}}^{1}\right) \cap B(x, r), B(x, 2 r)\right)}{\mu(B(x, r))}>0 .
$$

According to Corollary 3.11, the set $F$ also satisfies

$$
\lim _{r \rightarrow 0} r \frac{P(F, B(x, r))}{\mu(B(x, r))}=0 .
$$

Thus by (3.6) and the doubling property of $\mu$,

$$
\lim _{r \rightarrow 0} r \frac{\mathcal{H}\left(\partial^{1} I_{F} \cap B(x, 2 r)\right)}{\mu(B(x, r))}=0
$$

Combining the fact that $x \in O_{E}$ with (4.3) and (4.4), we find a number $a>0$ and a radius

$$
0<r_{f} \leq \frac{\min \left\{R, s_{1}\right\}}{2}
$$

such that

$$
\frac{\mu\left(E \cap B\left(x, 2 r_{f}\right)\right)}{\mu\left(B\left(x, 2 r_{f}\right)\right)} \leq \frac{1}{2 C_{d}^{\left\lceil\log _{2}(60 \lambda)\right\rceil}}
$$

and

$$
r_{f} \frac{\operatorname{cap}_{1}\left(\left(I_{E} \backslash{\overline{I_{F}}}^{1}\right) \cap B\left(x, r_{f}\right), B\left(x, 2 r_{f}\right)\right)}{\mu\left(B\left(x, r_{f}\right)\right)}>a
$$

and

$$
r_{f} \frac{\mathcal{H}\left(\partial^{1} I_{F} \cap B\left(x, 2 r_{f}\right)\right)}{\mu\left(B\left(x, r_{f}\right)\right)}<\frac{a}{16 C_{d}^{\left\lceil\log _{2}(10 \lambda)\right\rceil+2} C_{P}}
$$

Step 2. Let $D$ consist of all points $z \in B\left(x, r_{f}\right) \backslash{\overline{I_{F}}}^{1}$ for which there exists a radius $0<t \leq(10 \lambda)^{-1} r_{f}$ such that

$$
\frac{\mu(F \cap B(z, t))}{\mu(B(z, t))}>\frac{1}{4 C_{d}}
$$

Consider $z \in D$ and the corresponding radius $t$. Since $z \notin{\overline{I_{F}}}^{-1} \supset I_{F} \cup \partial^{*} F$ (recall $(3.5)$ ), we have

$$
\lim _{r \rightarrow 0} \frac{\mu(F \cap B(z, r))}{\mu(B(z, r))}=0 .
$$


Take the smallest $k=0,1, \ldots$ such that

$$
\frac{\mu\left(F \cap B\left(z, 2^{-k} t\right)\right)}{\mu\left(B\left(z, 2^{-k} t\right)\right)} \leq \frac{1}{2} .
$$

If $k=0$, let $r_{z}:=t$ so that

$$
\frac{1}{4 C_{d}}<\frac{\mu\left(F \cap B\left(z, r_{z}\right)\right)}{\mu\left(B\left(z, r_{z}\right)\right)} \leq \frac{1}{2}
$$

If $k \geq 1$, let $r_{z}:=2^{-k+1} t$, and then

$$
\frac{\mu\left(F \cap B\left(z, r_{z}\right)\right)}{\mu\left(B\left(z, r_{z}\right)\right)}>\frac{1}{2}
$$

and

$$
\begin{aligned}
\frac{\mu\left(F \cap B\left(z, r_{z}\right)\right)}{\mu\left(B\left(z, r_{z}\right)\right)} & =\frac{\mu\left(F \cap B\left(z, 2^{-k+1} t\right)\right)}{\mu\left(B\left(z, 2^{-k+1} t\right)\right)} \\
& \leq \frac{\mu\left(B\left(z, 2^{-k+1} t\right)\right)-\mu\left(B\left(z, 2^{-k} t\right) \backslash F\right)}{\mu\left(B\left(z, 2^{-k+1} t\right)\right)} \\
& \leq \frac{\mu\left(B\left(z, 2^{-k+1} t\right)\right)-\mu\left(B\left(z, 2^{-k} t\right)\right) / 2}{\mu\left(B\left(z, 2^{-k+1} t\right)\right)} \text { by (4.9) } \\
& \leq 1-\frac{1}{2 C_{d}} .
\end{aligned}
$$

Thus in both cases, we have $r_{z} \leq(10 \lambda)^{-1} r_{f}$ and

$$
\frac{1}{4 C_{d}}<\frac{\mu\left(F \cap B\left(z, r_{z}\right)\right)}{\mu\left(B\left(z, r_{z}\right)\right)} \leq 1-\frac{1}{2 C_{d}} .
$$

By the relative isoperimetric inequality (3.7) we now obtain

$$
\mu\left(B\left(z, r_{z}\right)\right) \leq 8 C_{d}^{2} C_{P} r_{z} \mathcal{H}\left(\partial^{1} I_{F} \cap B\left(x, \lambda r_{z}\right)\right) .
$$

Performing the same for every $z \in D$, we obtain a covering $\left\{B\left(z, \lambda r_{z}\right)\right\}_{z \in D}$. By the 5 -covering theorem, we can extract a countable collection $\left\{B_{j}=B\left(z_{j}, r_{j}\right)\right\}_{j=1}^{\infty}$ such that the balls $\lambda B_{j}$ are pairwise disjoint and $D \subset \bigcup_{j=1}^{\infty} 5 \lambda B_{j}$. For each $j \in \mathbb{N}$, define the Lipschitz function

$$
\eta_{j}:=\max \left\{0,1-\frac{\operatorname{dist}\left(\cdot, 5 \lambda B_{j}\right)}{5 \lambda r_{j}}\right\},
$$

so that $\eta_{j}=1$ on $5 \lambda B_{j}, \eta_{j}=0$ outside $10 \lambda B_{j}$, and the minimal 1-weak upper gradient satisfies $g_{\eta_{j}} \leq\left(5 \lambda r_{j}\right)^{-1} \chi_{10 \lambda B_{j}}$ (see [5, Corollary 2.21]). Moreover, $r_{j} \leq$ 
$(10 \lambda)^{-1} r_{f}$ and so $\eta_{j} \in N_{0}^{1,1}\left(B\left(x, 2 r_{f}\right)\right)$ for all $j \in \mathbb{N}$. Now we have

$$
\begin{aligned}
\operatorname{cap}_{1}\left(D, B\left(x, 2 r_{f}\right)\right) & \leq \operatorname{cap}_{1}\left(\bigcup_{j=1}^{\infty} 5 \lambda B_{j}, B\left(x, 2 r_{f}\right)\right) \\
& \leq \sum_{j=1}^{\infty} \operatorname{cap}_{1}\left(5 \lambda B_{j}, B\left(x, 2 r_{f}\right)\right) \\
& \leq \sum_{j=1}^{\infty} \int_{X} g_{\eta_{j}} d \mu \\
& \leq \sum_{j=1}^{\infty} \frac{\mu\left(10 \lambda B_{j}\right)}{5 \lambda r_{j}} \\
& \leq C_{d}^{\left\lceil\log _{2}(10 \lambda)\right\rceil} \sum_{j=1}^{\infty} \frac{\mu\left(B_{j}\right)}{r_{j}} \\
& \leq 8 C_{d}^{\left\lceil\log _{2}(10 \lambda)\right\rceil+2} C_{P} \sum_{j=1}^{\infty} \mathcal{H}\left(\partial^{1} I_{F} \cap \lambda B_{j}\right) \quad \text { by (4.10) } \\
& \leq 8 C_{d}^{\left\lceil\log _{2}(10 \lambda)\right\rceil+2} C_{P} \mathcal{H}\left(\partial^{1} I_{F} \cap B\left(x, 2 r_{f}\right)\right) .
\end{aligned}
$$

Thus by (4.8),

$$
r_{f} \frac{\operatorname{cap}_{1}\left(D, B\left(x, 2 r_{f}\right)\right)}{\mu\left(B\left(x, r_{f}\right)\right)}<\frac{a}{2}
$$

and so

$$
\begin{aligned}
& r_{f} \frac{\operatorname{cap}_{1}\left(\left(I_{E} \backslash\left({\overline{I_{F}}}^{1} \cup D\right)\right) \cap B\left(x, r_{f}\right), B\left(x, 2 r_{f}\right)\right)}{\mu\left(B\left(x, r_{f}\right)\right)} \\
& \geq r_{f} \frac{\operatorname{cap}_{1}\left(\left(I_{E} \backslash{\overline{I_{F}}}^{1}\right) \cap B\left(x, r_{f}\right), B\left(x, 2 r_{f}\right)\right)-\operatorname{cap}_{1}\left(D, B\left(x, 2 r_{f}\right)\right)}{\mu\left(B\left(x, r_{f}\right)\right)} \\
& >\frac{a}{2}
\end{aligned}
$$

by (4.7).

Step 3. Now consider $z \in\left(I_{E} \backslash\left({\overline{I_{F}}}^{1} \cup D\right)\right) \cap B\left(x, r_{f}\right)$. We have

$$
\lim _{r \rightarrow 0} \frac{\mu(E \cap B(z, r))}{\mu(B(z, r))}=1,
$$

and so we can choose $0<t \leq(20 \lambda)^{-1} r_{f}$ such that

$$
\frac{\mu(E \cap B(z, t))}{\mu(B(z, t))}>\frac{1}{2} .
$$


Note also that for any $r \in\left[(20 \lambda)^{-1} r_{f},(10 \lambda)^{-1} r_{f}\right]$, we have $B\left(x, 2 r_{f}\right) \subset B(z, 60 \lambda r)$ and so

$$
\frac{\mu(E \cap B(z, r))}{\mu(B(z, r))} \leq C_{d}^{\left\lceil\log _{2}(60 \lambda)\right\rceil} \frac{\mu\left(E \cap B\left(x, 2 r_{f}\right)\right)}{\mu\left(B\left(x, 2 r_{f}\right)\right)} \leq \frac{1}{2}
$$

by (4.6). Set $r_{z}:=2^{k} t$ for the smallest $k \in \mathbb{N}$ such that

$$
\frac{\mu\left(E \cap B\left(z, 2^{k} t\right)\right)}{\mu\left(B\left(z, 2^{k} t\right)\right)} \leq \frac{1}{2}
$$

Then we have $0<r_{z} \leq(10 \lambda)^{-1} r_{f}$ and

$$
\frac{1}{2 C_{d}}<\frac{\mu\left(E \cap B\left(z, r_{z}\right)\right)}{\mu\left(B\left(z, r_{z}\right)\right)} \leq \frac{1}{2}
$$

and since $z \notin D$,

$$
\frac{1}{4 C_{d}} \leq \frac{\mu\left((E \backslash F) \cap B\left(z, r_{z}\right)\right)}{\mu\left(B\left(z, r_{z}\right)\right)} \leq \frac{1}{2} .
$$

Then by the relative isoperimetric inequality (3.7), we have

$$
\frac{\mu\left(B\left(z, r_{z}\right)\right)}{r_{z}} \leq 8 C_{d}^{2} C_{P} \mathcal{H}\left(\partial^{1} I_{E \backslash F} \cap B\left(z, \lambda r_{z}\right)\right) .
$$

(Note that the right-hand side could be infinity.) Let

$$
A:=\bigcup_{z \in\left(I_{E} \backslash\left({\overline{I_{F}}}^{1} \cup D\right)\right) \cap B\left(x, r_{f}\right)} B\left(z, \lambda r_{z}\right) \subset B\left(x, 2 r_{f}\right) .
$$

Consider the covering $\left\{B\left(z, \lambda r_{z}\right)\right\}_{z \in\left(I_{E} \backslash\left(\bar{I}_{F}^{1} \cup D\right)\right) \cap B\left(x, r_{f}\right)}$. By the 5-covering theorem, we can extract a countable collection $\left\{B_{j}=B\left(z_{j}, r_{j}\right)\right\}_{j=1}^{\infty}$ such that the balls $\lambda B_{j}$ are pairwise disjoint and $\left(I_{E} \backslash\left(\bar{I}_{F}^{1} \cup D\right)\right) \cap B\left(x, r_{f}\right) \subset \bigcup_{j=1}^{\infty} 5 \lambda B_{j}$. Just as in the previous step, for each $j \in \mathbb{N}$ define the Lipschitz function

$$
\eta_{j}:=\max \left\{0,1-\frac{\operatorname{dist}\left(\cdot, 5 \lambda B_{j}\right)}{5 \lambda r_{j}}\right\},
$$

so that $\eta_{j}=1$ on $5 \lambda B_{j}, \eta_{j}=0$ outside $10 \lambda B_{j}$, and the minimal 1-weak upper gradient satisfies $g_{\eta_{j}} \leq\left(5 \lambda r_{j}\right)^{-1} \chi_{10 \lambda B_{j}}$. Moreover, $r_{j} \leq(10 \lambda)^{-1} r_{f}$ and so $\eta_{j} \in$ 
$N_{0}^{1,1}\left(B\left(x, 2 r_{f}\right)\right)$ for all $j \in \mathbb{N}$. Now we have

$$
\begin{aligned}
& \operatorname{cap}_{1}\left(\left(I_{E} \backslash\left({\overline{I_{F}}}^{1} \cup D\right)\right) \cap B\left(x, r_{f}\right), B\left(x, 2 r_{f}\right)\right) \\
& \leq \operatorname{cap}_{1}\left(\bigcup_{j=1}^{\infty} 5 \lambda B_{j}, B\left(x, 2 r_{f}\right)\right) \\
& \leq \sum_{j=1}^{\infty} \operatorname{cap}_{1}\left(5 \lambda B_{j}, B\left(x, 2 r_{f}\right)\right) \\
& \leq \sum_{j=1}^{\infty} \int_{X} g_{\eta_{j}} d \mu \\
& \leq \sum_{j=1}^{\infty} \frac{\mu\left(10 \lambda B_{j}\right)}{5 \lambda r_{j}} \\
& \leq C_{d}^{\left\lceil\log _{2}(10 \lambda)\right\rceil} \sum_{j=1}^{\infty} \frac{\mu\left(B_{j}\right)}{r_{j}} \\
& \leq 8 C_{d}^{\left\lceil\log _{2}(10 \lambda)\right\rceil+2} C_{P} \sum_{j=1}^{\infty} \mathcal{H}\left(\partial^{1} I_{E \backslash F} \cap \lambda B_{j}\right) \quad \text { by (4.13) } \\
& \leq 8 C_{d}^{\left\lceil\log _{2}(10 \lambda)\right\rceil+2} C_{P} \mathcal{H}\left(\partial^{1} I_{E \backslash F} \cap A\right) .
\end{aligned}
$$

Step 4. Next we show that

$$
\partial^{1} I_{E \backslash F} \subset\left(\partial^{1} I_{E} \backslash{\overline{I_{F}}}^{1}\right) \cup \partial^{1} I_{F} .
$$

To see this, note that $X \backslash{\overline{I_{F}}}^{1} \subset O_{F}$ (recall (3.5)) and so $I_{E \backslash F} \backslash{\overline{I_{F}}}^{1}=I_{E} \backslash{\overline{I_{F}}}^{1}$. Since $X \backslash{\overline{I_{F}}}^{1}$ is a 1-finely open set, it follows that $\partial^{1} I_{E \backslash F} \backslash{\overline{I_{F}}}^{1}=\partial^{1} I_{E} \backslash{\overline{I_{F}}}^{1}$. Moreover, $I_{E \backslash F} \cap$ fine-int $I_{F}=\emptyset$ and fine-int $I_{F}$ is 1-finely open, and so $\partial^{1} I_{E \backslash F} \cap$ fine-int $I_{F}=\emptyset$. From these, (4.16) follows.

By (4.11) and (4.15),

$$
r_{f} \frac{\mathcal{H}\left(\partial^{1} I_{E \backslash F} \cap A\right)}{\mu\left(B\left(x, r_{f}\right)\right)} \geq \frac{a}{16 C_{d}^{\left\lceil\log _{2}(10 \lambda)\right\rceil+2} C_{P}} .
$$

Now by first using (4.16) and the fact that $A \subset B\left(x, 2 r_{f}\right)$ (recall (4.14)), and then (4.8) and the above inequality, we get

$$
r_{f} \frac{\mathcal{H}\left(\left(\partial^{1} I_{E} \backslash{\overline{I_{F}}}^{1}\right) \cap A\right)}{\mu\left(B\left(x, r_{f}\right)\right)} \geq r_{f} \frac{\mathcal{H}\left(\partial^{1} I_{E \backslash F} \cap A\right)-\mathcal{H}\left(\partial^{1} I_{F} \cap B\left(x, 2 r_{f}\right)\right)}{\mu\left(B\left(x, r_{f}\right)\right)}>0 .
$$

In particular, there exists a point $y \in\left(\partial^{1} I_{E} \backslash \bar{I}_{F}^{1}\right) \cap A$. Recall from (4.5) that $0<r_{f} \leq \min \left\{R, s_{1}\right\} / 2$, and so $\bar{W}^{1} \cap B\left(x, 2 r_{f}\right) \subset I_{F} \cap B\left(x, 2 r_{f}\right)$. Thus

$$
y \in\left(\partial^{1} I_{E} \backslash \bar{W}^{1}\right) \cap B\left(x, 2 r_{f}\right) \subset\left(\partial^{1} I_{E} \backslash \bar{W}^{1}\right) \cap B\left(x, s_{1}\right),
$$


as desired. By the definition of $A$ (recall (4.12), (4.14) ) there is a point $z \in X$ and a radius $0<r_{z} \leq(10 \lambda)^{-1} r_{f}$ such that $y \in B\left(z, \lambda r_{z}\right)$ and

$$
\frac{1}{2 C_{d}} \leq \frac{\mu\left(E \cap B\left(z, r_{z}\right)\right)}{\mu\left(B\left(z, r_{z}\right)\right)} \leq \frac{1}{2}
$$

For $s_{2}:=2 \lambda r_{z} \leq s_{1} / 2$ we then have $B\left(z, r_{z}\right) \subset B\left(y, s_{2}\right) \subset B\left(z, 3 \lambda r_{z}\right)$, and so

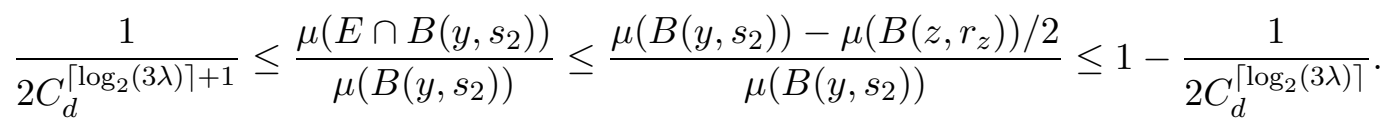

This completes the proof of the claim.

Define $r_{0}=1$. We use the claim repeatedly, first with the choice $x=x_{0}$ and $s_{1}=r_{0}$, to find a sequence of points $x_{j} \in B\left(x_{j-1}, r_{j-1}\right) \cap \partial^{1} I_{E} \backslash \bar{W}^{1}$ and a sequence of numbers $0<r_{j} \leq r_{j-1} / 2$ such that

$$
\min \left\{\frac{\mu\left(B\left(x_{j}, r_{j}\right) \cap E\right)}{\mu\left(B\left(x_{j}, r_{j}\right)\right)}, \frac{\mu\left(B\left(x_{j}, r_{j}\right) \backslash E\right)}{\mu\left(B\left(x_{j}, r_{j}\right)\right)}\right\} \geq \frac{1}{2 C_{d}^{\left\lceil\log _{2}(3 \lambda)\right\rceil+1}}
$$

for all $j \in \mathbb{N}$. By completeness of the space and the fact that $W$ is open, we find $x \in X \backslash W$ such that $x_{j} \rightarrow x$. For each $j \in \mathbb{N}$ we have

$$
d\left(x, x_{j}\right) \leq \sum_{k=j}^{\infty} d\left(x_{k}, x_{k+1}\right) \leq \sum_{k=j}^{\infty} r_{k} \leq 2 r_{j}
$$

Thus $B\left(x_{j}, r_{j}\right) \subset B\left(x, 3 r_{j}\right) \subset B\left(x_{j}, 5 r_{j}\right)$ for all $j \in \mathbb{N}$, and so

$$
\frac{\mu\left(B\left(x, 3 r_{j}\right) \cap E\right)}{\mu\left(B\left(x, 3 r_{j}\right)\right)} \geq \frac{\mu\left(B\left(x_{j}, r_{j}\right) \cap E\right)}{\mu\left(B\left(x, 3 r_{j}\right)\right)} \geq \frac{1}{C_{d}^{3}} \frac{\mu\left(B\left(x_{j}, r_{j}\right) \cap E\right)}{\mu\left(B\left(x_{j}, r_{j}\right)\right)} \geq \frac{1}{2 C_{d}^{\left\lceil\log _{2}(3 \lambda)\right\rceil+4}}
$$

and similarly

$$
\frac{\mu\left(B\left(x, 3 r_{j}\right) \backslash E\right)}{\mu\left(B\left(x, 3 r_{j}\right)\right)} \geq \frac{\mu\left(B\left(x_{j}, r_{j}\right) \backslash E\right)}{\mu\left(B\left(x, 3 r_{j}\right)\right)} \geq \frac{1}{C_{d}^{3}} \frac{\mu\left(B\left(x_{j}, r_{j}\right) \backslash E\right)}{\mu\left(B\left(x_{j}, r_{j}\right)\right)} \geq \frac{1}{2 C_{d}^{\left\lceil\log _{2}(3 \lambda)\right\rceil+4}} .
$$

Thus

$$
\limsup _{r \rightarrow 0} \frac{\mu(B(x, r) \cap E)}{\mu(B(x, r))}>0 \quad \text { and } \quad \limsup _{r \rightarrow 0} \frac{\mu(B(x, r) \backslash E)}{\mu(B(x, r))}>0,
$$

and so $x \in \partial^{*} E \backslash W$, which proves the theorem by the discussion in the first paragraph of the proof.

By using another argument involving Lipschitz cutoff functions, it is easy to see that for any $A \subset X$ and any ball $B(x, r)$,

$$
\operatorname{cap}_{1}(A \cap B(x, r), B(x, 2 r)) \leq C_{d} \mathcal{H}(A \cap B(x, r)) .
$$

Theorem 4.18. Let $\Omega \subset X$ be open and let $E \subset X$ be $\mu$-measurable with $\mathcal{H}\left(\partial^{*} E \cap\right.$ $\Omega)<\infty$. Then $\mathcal{H}\left(\left(\partial^{1} I_{E} \backslash \partial^{*} E\right) \cap \Omega\right)=0$. 
Proof. By a standard covering argument (see e.g. the proof of [17, Lemma 2.6]) we find that

$$
\lim _{r \rightarrow 0} r \frac{\mathcal{H}\left(\partial^{*} E \cap B(x, r)\right)}{\mu(B(x, r))}=0
$$

for all $x \in \Omega \backslash\left(\partial^{*} E \cup N\right)$, with $\mathcal{H}(N)=0$. Then by (4.17), also

$$
\begin{aligned}
\limsup _{r \rightarrow 0} r \frac{\operatorname{cap}_{1}\left(\left(\partial^{*} E \cup N\right) \cap B(x, r), B(x, 2 r)\right)}{\mu(B(x, r))} & \leq C_{d} \limsup _{r \rightarrow 0} r \frac{\mathcal{H}\left(\left(\partial^{*} E \cup N\right) \cap B(x, r)\right)}{\mu(B(x, r))} \\
& =C_{d} \limsup _{r \rightarrow 0} r \frac{\mathcal{H}\left(\partial^{*} E \cap B(x, r)\right)}{\mu(B(x, r))} \\
& =0
\end{aligned}
$$

for all $x \in \Omega \backslash\left(\partial^{*} E \cup N\right)$. Thus $\Omega \backslash\left(\partial^{*} E \cup N\right)$ is a 1-finely open set. Now by Theorem 4.1. $\partial^{1} I_{E} \cap\left(\Omega \backslash\left(\partial^{*} E \cup N\right)\right)=\emptyset$ and the result follows.

Now we can prove our main theorem.

Proof of Theorem 1.1. By Theorem 4.18 we have $\mathcal{H}\left(\partial^{1} I_{E} \cap \Omega\right)<\infty$. Then by Theorem 1.2 we have $P(E, \Omega)<\infty$.

\section{Some consequences and discussion}

Now we can state Federer's characterization in metric spaces as follows.

Corollary 5.1. Let $\Omega \subset X$ be open and let $E \subset X$ be $\mu$-measurable. Then $P(E, \Omega)<\infty$ if and only if $\mathcal{H}\left(\partial^{*} E \cap \Omega\right)<\infty$.

Proof. This follows by combining Theorem 1.1 and (2.4).

In general, the sets $\partial^{*} E$ and $\partial^{1} I_{E}$ can be quite different.

Example 5.2. Let $X=\mathbb{R}$ (unweighted). Let $\left\{q_{j}\right\}_{j=1}^{\infty}$ be an enumeration of all rational numbers and let $E:=\bigcup_{j=1}^{\infty} B\left(q_{j}, 2^{-j}\right)$. Then $\mathcal{L}^{1}\left(I_{E}\right) \leq 2$ and $\mathcal{L}^{1}\left(\partial^{*} E\right)=0$ by Lebesgue's differentiation theorem. On the other hand, it is straightforward to check that for any $A \subset \mathbb{R}$, we have $\partial^{1} A=\partial A$. Thus $\partial^{1} I_{E}=\partial I_{E} \supset \mathbb{R} \backslash I_{E}$ and so $\mathcal{L}^{1}\left(\partial^{1} I_{E}\right)=\infty$.

In the Euclidean setting, the "if" direction of Federer's characterization is proved by first showing that almost every coordinate line intersecting $I_{E}$ and $O_{E}$ also intersects $\partial^{*} E$, see [11, Section 4.5.11] or [10, p. 222-]. Proving this fact relies heavily on the Euclidean structure, and so it is difficult to generalize to metric spaces. However, we do have the following; it would be interesting to know if the assumption $\mathcal{H}\left(\partial^{*} E\right)<\infty$ can be dropped.

Proposition 5.3. Let $E \subset X$ be $\mu$-measurable and suppose that $\mathcal{H}\left(\partial^{*} E\right)<\infty$. Then 1-almost every curve intersecting $I_{E}$ and $O_{E}$ also intersects $\partial^{*} E$.

Proof. By Theorem 1.1, $P(E, X)<\infty$. Then the result follows from [25, Corollary $6.4]$. 
It is reasonable to expect Federer's characterization to find various applications especially in the metric setting, where certain tools of Euclidean BV theory, such as the Gauss-Green theorem, are not available. One likely application is in the study of images of sets of finite perimeter under quasiconformal mappings (see 15] for the Euclidean case), since such mappings are known to preserve the measure-theoretic boundary (see [20, Theorem 6.1]).

Now we discuss some existing applications. From the characterization it follows that the space supports the following strong relative isoperimetric inequality introduced in [16]; compare this with (2.2) and (3.7).

Corollary 5.4. For every ball $B(x, r)$ and every $\mu$-measurable $E \subset X$, we have

$$
\min \{\mu(B(x, r) \cap E), \mu(B(x, r) \backslash E)\} \leq 2 C_{P} C_{d} r \mathcal{H}\left(\partial^{*} E \cap B(x, \lambda r)\right) .
$$

Proof. We can assume that the right-hand side is finite. By Theorem 1.1 we know that $P(E, B(x, \lambda r))<\infty$, and now the result follows by combining the relative isoperimetric inequality (2.2) and (2.4).

In [16] the authors worked with the same standing assumptions as we do in the current paper, but additionally they assumed that the space supports the above strong relative isoperimetric inequality. Now we know that this does not need to be separately assumed, and the following theorem (Theorem 1.1 of [16]) holds under our standing assumptions (completeness, doubling, and Poincaré).

Theorem 5.5. Let $\Omega \subset X$ be a bounded open set and let $u \in N^{1, p}(\Omega)$ with $1 \leq p<$ $\infty$. Then $u \in N_{0}^{1, p}(\Omega)$ if and only if

$$
\lim _{r \rightarrow 0} \frac{1}{\mu(B(x, r))} \int_{\Omega \cap B(x, r)}|u| d \mu=0
$$

for $\mathrm{Cap}_{p}$-almost every $x \in \partial \Omega$.

Theorem 6.1 in [26] considered an analogous characterization of a class of BV functions with zero boundary values, also under the additional assumption of a strong relative isoperimetric inequality. Such a class is needed in an ongoing study of new fine properties of BV functions and capacities (begun in [24]), and this was in fact a key motivation for the current paper. The strong relative isoperimetric inequality was also used in proving approximation results for BV functions, see [26, Corollary 6.7, Theorem 6.9] as well as [25, Corollary 7.6] and the comment after it. Now we know that all of these results hold in every complete metric space equipped with a doubling measure and supporting a Poincaré inequality.

\section{References}

[1] D. Adams and L. I. Hedberg, Function spaces and potential theory, Grundlehren der Mathematischen Wissenschaften, 314. Springer-Verlag, Berlin, 1996. xii+366 pp. 
[2] L. Ambrosio, Fine properties of sets of finite perimeter in doubling metric measure spaces, Calculus of variations, nonsmooth analysis and related topics. Set-Valued Anal. 10 (2002), no. 2-3, 111-128.

[3] L. Ambrosio, N. Fusco, and D. Pallara, Functions of bounded variation and free discontinuity problems. Oxford Mathematical Monographs. The Clarendon Press, Oxford University Press, New York, 2000.

[4] L. Ambrosio, M. Miranda, Jr., and D. Pallara, Special functions of bounded variation in doubling metric measure spaces, Calculus of variations: topics from the mathematical heritage of E. De Giorgi, 1-45, Quad. Mat., 14, Dept. Math., Seconda Univ. Napoli, Caserta, 2004.

[5] A. Björn and J. Björn, Nonlinear potential theory on metric spaces, EMS Tracts in Mathematics, 17. European Mathematical Society (EMS), Zürich, 2011. xii+403 pp.

[6] A. Björn and J. Björn, Obstacle and Dirichlet problems on arbitrary nonopen sets in metric spaces, and fine topology, Rev. Mat. Iberoam. 31 (2015), no. 1, 161-214.

[7] A. Björn, J. Björn, and V. Latvala, Sobolev spaces, fine gradients and quasicontinuity on quasiopen sets, Ann. Acad. Sci. Fenn. Math. 41 (2016), no. 2, 551-560.

[8] A. Björn, J. Björn, and V. Latvala, The Cartan, Choquet and Kellogg properties for the fine topology on metric spaces, to appear in J. Anal. Math.

[9] A. Björn, J. Björn, and V. Latvala, The weak Cartan property for the p-fine topology on metric spaces, Indiana Univ. Math. J. 64 (2015), no. 3, 915-941.

[10] L. C. Evans and R. F. Gariepy, Measure theory and fine properties of functions, Studies in Advanced Mathematics series, CRC Press, Boca Raton, 1992.

[11] H. Federer, Geometric measure theory, Die Grundlehren der mathematischen Wissenschaften, Band 153 Springer-Verlag New York Inc., New York 1969 xiv+676 pp.

[12] E. Giusti, Minimal surfaces and functions of bounded variation, Monographs in Mathematics, 80. Birkhäuser Verlag, Basel, 1984. xii+240 pp.

[13] J. Heinonen, T. Kilpeläinen, and O. Martio, Nonlinear potential theory of degenerate elliptic equations, Unabridged republication of the 1993 original. Dover Publications, Inc., Mineola, NY, 2006. xii+404 pp.

[14] J. Heinonen and P. Koskela, Quasiconformal maps in metric spaces with controlled geometry, Acta Math. 181 (1998), no. 1, 1-61. 
[15] J. Kelly, Quasiconformal mappings and sets of finite perimeter, Trans. Amer. Math. Soc. 180 (1973), 367-387.

[16] J. Kinnunen, R. Korte, N. Shanmugalingam, and H. Tuominen, A characterization of Newtonian functions with zero boundary values, Calc. Var. Partial Differential Equations 43 (2012), no. 3-4, 507-528.

[17] J. Kinnunen, R. Korte, N. Shanmugalingam, and H. Tuominen, Pointwise properties of functions of bounded variation in metric spaces, Rev. Mat. Complut. 27 (2014), no. 1, 41-67.

[18] R. Korte and P. Lahti, Relative isoperimetric inequalities and sufficient conditions for finite perimeter on metric spaces, Ann. Inst. H. Poincaré Anal. Non Linéaire 31 (2014), no. 1, 129-154.

[19] R. Korte, P. Lahti, and N. Shanmugalingam, Semmes family of curves and a characterization of functions of bounded variation in terms of curves, Calc. Var. Partial Differential Equations 54 (2015), no. 2, 1393-1424.

[20] R. Korte, N. Marola, and N. Shanmugalingam, Quasiconformality, homeomorphisms between metric measure spaces preserving quasiminimizers, and uniform density property, Ark. Mat. 50 (2012), no. 1, 111-134.

[21] P. Lahti, A Federer-style characterization of sets of finite perimeter on metric spaces, Calc. Var. Partial Differential Equations 56 (2017), no. 5, Art. 150, 22 pp.

[22] P. Lahti, A notion of fine continuity for BV functions on metric spaces, Potential Anal. 46 (2017), no. 2, 279-294.

[23] P. Lahti, Superminimizers and a weak Cartan property for $p=1$ in metric spaces, to appear in J. Anal. Math.

[24] P. Lahti, The variational 1-capacity and BV functions with zero boundary values on metric spaces, preprint 2017. https://arxiv.org/abs/1708.09318

[25] P. Lahti and N. Shanmugalingam, Fine properties and a notion of quasicontinuity for $B V$ functions on metric spaces, J. Math. Pures Appl. (9) 107 (2017), no. 2, 150-182.

[26] P. Lahti and N. Shanmugalingam, Trace theorems for functions of bounded variation in metric spaces, to appear in Journal of Functional Analysis.

[27] J. Lukeš, J. Malý, and L. Zajíček, Fine topology methods in real analysis and potential theory, Lecture Notes in Mathematics, 1189. SpringerVerlag, Berlin, 1986. x+472 pp.

[28] J. Malý and W. Ziemer, Fine regularity of solutions of elliptic partial differential equations, Mathematical Surveys and Monographs, 51. American Mathematical Society, Providence, RI, 1997. xiv+291 pp. 
[29] M. Miranda, Jr., Functions of bounded variation on "good" metric spaces, J. Math. Pures Appl. (9) 82 (2003), no. 8, 975-1004.

[30] N. Shanmugalingam, Newtonian spaces: An extension of Sobolev spaces to metric measure spaces, Rev. Mat. Iberoamericana 16(2) (2000), 243-279.

[31] W. P. Ziemer, Weakly differentiable functions. Sobolev spaces and functions of bounded variation, Graduate Texts in Mathematics, 120. SpringerVerlag, New York, 1989.

Address:

University of Jyvaskyla

Department of Mathematics and Statistics

P.O. Box 35, FI-40014 University of Jyvaskyla

E-mail: panu.k.lahti@jyu.fi 